\title{
Non-COVID Surgical Emergency During the Nationwide Lockdown due to Corona Pandemic: a Critical Appraisal
}

\author{
Rahul $^{1} \cdot$ Alka Verma $^{2} \cdot$ Priyank Yadav $^{3} \cdot$ Vijay Kumar Sharma $^{1} \cdot$ Om Prakash Sanjeev ${ }^{2}$
}

Received: 8 June 2020 / Accepted: 29 July 2020 / Published online: 10 August 2020

(C) Association of Surgeons of India 2020

\begin{abstract}
The World Health Organization (WHO) declared corona infection as a pandemic in February 2020. A nationwide lockdown was enforced by Indian government on 25 March 2020. Separate health facilities were developed to handle the confirmed and suspected cases of COVID-19 (coronavirus disease). Other than emergency services and care of cancer patients, all remaining healthcare activities were curtailed. Through this study, we intend to assess any change in number and pattern of non-COVID surgical emergencies during the lockdown as well as the interventions required. This was an observational study which included all patients with surgical emergencies who presented during the study period (25 March to 24 April 2020) after two stage screenings for corona infection (group 2). The results obtained from analysis of prospectively collected database were compared with a similar period (group 1) prior to the onset of pandemic in India using appropriate statistical tests. In group 2, an increase $(17 \%)$ in number of patients was noted. The need of organ support was more than 4 times the usual period. An upsurge in neurosurgical emergencies was noted, though the number of interventions decreased by $40 \%$. A significant decrease in hospital stay was also documented ( 7 days vs 12 days). The nationwide lockdown led to an increase and change in pattern of surgical emergencies, though the interventions required were less. Effective management entails appropriate preparedness.
\end{abstract}

Keywords COVID- $19 \cdot$ Corona $\cdot$ Surgical emergency $\cdot$ Non-COVID $\cdot$ Lockdown $\cdot$ Pandemic

\section{Introduction}

An outbreak of viral infection emerged in China in December 2019. A novel coronavirus named COVID-19 (coronavirus disease) was isolated as the causative agent. Despite containment measures taken by China and other countries, the case count soared so high that the disease was declared a pandemic by the World Health Organization (WHO) in February 2020 [1]. The first case in India was recorded on 30 January 2020 in the southern state of Kerala. The virus spreads through direct contact, fomites, and droplets [2]. A slow but steady increase

Alka Verma

dralka.verma91@gmail.com

1 Department of Surgical Gastroenterology, Sanjay Gandhi Post Graduate Institute of Medical Sciences, Lucknow, India

2 Department of Emergency Medicine, Sanjay Gandhi Post Graduate Institute of Medical Sciences, Raibareli Road, Lucknow, India

3 Department of Urology and Renal Transplantation, Sanjay Gandhi Post Graduate Institute of Medical Sciences, Lucknow, India in the number of cases throughout the country was noted in the months of February and March. To prevent the exponential growth and a sudden outburst of patients, a nationwide lockdown was enforced by the Indian government from 25 March 2020, when the case count in the country was 500 . The primary intention was social distancing to put a check on the transmission and buy time for preparedness. As compared with the Western countries, the early enforcement of lockdown in India diminished the initial rate of progression [3].The recorded patients of coronavirus and mortality associated with the disease remained substantially low as compared with European and American countries.

In order to ensure that the focus of the healthcare system remains undivided in the fight against coronavirus, except for emergency services and care of cancer patients, all remaining healthcare activities were curtailed during the lockdown. The outpatient department activities were put on hold (other than malignancy and end-stage organ disease). Hospital admissions and elective surgeries were postponed to safeguard the manpower and resources. The essential emergency services remained functional. At many centers, a separate health facility was created within a span of 1-2 weeks to handle the 
confirmed and suspected cases of COVID-19 [4]. The response to coronavirus outbreak in the province of Uttar Pradesh with a population of over 200 million was swift, and as the lockdown was enforced, a 3-tier triage system was introduced [4]. The trauma cases during the lockdown were expected to be low. The trauma center at our institute was converted to a corona hospital within 2 weeks. All nonCOVID emergency services continued to be delivered by the Department of Emergency Medicine in the main hospital.

During the lockdown period in India, the services to patients who were not at imminent risk took a serious toll [5]. The lack of standard guidelines and dearth of eloquent personal protection equipment for screening and management of patients in the first month impeded the healthcare services offered by the nursing homes and private practitioners, a trend that mimicked the one seen in Italy earlier [6]. As a result, many patients who would not normally come to the emergency department at a government center were forced to do so. The increased load on emergency during a contagious pandemic conjures efficient screening system of COVID suspects and triage to bestow maximum benefit to the patients. Organizing separate traffics for the epidemic patients and non-COVID casualties entails huge redistribution of manpower. In the period of crisis, it is prudent to judiciously utilize the limited resources. The available staff members need to be trained and reorganized to manage increasing number of concurrent emergencies. With limited number of operation theaters and available anesthesia support, it is pragmatic to learn about changes in number and type of surgical emergencies. This will aid in formulating effective plans. Through this study, we intend to highlight the difference in patterns of patients who presented as a surgical emergency during the lockdown period (COVID outbreak).

The primary end points of the study were (a) to assess any change in number and pattern of patients with surgical emergency during the lockdown and (b) to assess the impact of lockdown on the duration of symptoms before reaching the emergency. The secondary endpoint of the study was to assess any change in the number of interventions.

\section{Patients and Methods}

This was an observational study conducted in the Department of Emergency Medicine at a tertiary care center in Northern India. Institute's Ethical Committee approval was obtained to conduct the study (IEC code: 2020-134-IP-EXP-20). The emergency department (ED) has 30 beds which cater to both surgical and medical emergencies. During the lockdown period, the patients were admitted to the department only after thorough screening with questionnaire (symptoms and travel history) and temperature probes for suspicion of corona. The suspected cases were evaluated at corona center in a holding area. Patients who were not suspected to be positive for corona and hailed from a low transmission area were directly admitted at the ED in the main hospital. All patients were tested for corona before any radiological or surgical intervention or before being shifted to their respective wards. In this study, we intended to evaluate the patients with surgical emergencies visiting the ED (in the non-COVID hospital) during the first month of national lockdown (25 March 2020 to 24 April 2020). Patients who were brought dead were excluded. The data was collected retrospectively from the prospectively maintained hospital records. The management of the patients was not altered by the study.

The data of patients during the lockdown period was compared with the profile and outcomes of patients referred to the respective departments over a similar period (1 month) prior to the onset of the pandemic in India. Any change in the characteristics of patients and their management were recorded and compared using appropriate statistical tests.

Sample Size Estimation To detect the 0.8 effect size $(\geq 0.8$ effect size between two independent groups is considered large effect) between two independent groups, at minimum two-sided $95 \%$ confidence interval and $80 \%$ power of the study, calculated sample size of the two groups came out to be 26 each. In this study, we have included 29 patients in group 1 (pre lockdown) and 34 in group 2 (during lockdown). Sample size was estimated using software G Power version 3.1.9.2 (Düsseldorf University, Germany).

Statistical Analysis Normality of the continuous variables was tested, and a variable was considered normally distributed when $\mathrm{Z}$ score of the skewness was \pm 3.29 . Continuous variables were presented in mean \pm standard deviation/median (interquartile range), whereas categorical data was presented in frequency $(\%)$. To compare the mean, median, and proportions between two groups, independent sample $t$ test, MannWhitney $U$ test, and chi-square test/Fisher exact test were used respectively. Error bar graph was used to present the distribution of means. A $p$ value $<0.05$ was considered statistically significant. Statistical package for social sciences version-23 (SPSS-23, IBM, Chicago, USA) was used for the analysis.

\section{Results}

The differences in the profile and management of the patients (surgical emergencies) who were managed in the ED of the non-COVID hospital during the first month of the lockdown period and a similar period in the non-pandemic era (before January 30th in India) have been highlighted in Tables 1 and 2 . There was a marginal increase $(17 \%)$ in the total number of surgical emergencies during the lockdown period, and the average distance covered by the patients to reach the hospital 
Table 1 Distribution of demographic, clinical values, and outcomes between pre and during COVID-19 lockdown $(N=63)$

\begin{tabular}{llll}
\hline Variables & Group 1 $(n=29)$ & Group 2(n=34) & $p$ value \\
\hline Age (years) & 43.24 SD 16.03 & 45.38 SD 14.02 & $0.574 \#$ \\
Males (\%) & $19(65.5 \%)$ & $18(52.9 \%)$ & $0.312^{*}$ \\
Distance from LKO in kilometers (range) & $190(70-290)$ & $215(156-270)$ & $0.290 \$$ \\
Duration of stay in days (range) & $12(6.5-22.5)$ & $7(3-11)$ & $0.007 \$$ \\
Duration of symptom in days (range) & $5(3-20)$ & $6(3-20)$ & $0.994 \$$ \\
Departments & & & \\
SGE & $26(89.7 \%)$ & $24(70.6 \%)$ & $0.110^{*}$ \\
URO & $3(10.3 \%)$ & $3(8.8 \%)$ & $0.999^{*}$ \\
NS & 0 & $7(20.6 \%)$ & $0.001^{*}$ \\
Patient type (new) & $20(69 \%)$ & $23(67.6 \%)$ & $0.911^{*}$ \\
Death & $2(6.9 \%)$ & $1(2.9 \%)$ & $0.590^{*}$ \\
LAMA & $2(6.9 \%)$ & $5(14.7 \%)$ & $0.436^{*}$ \\
Discharge/transfer to respective units & $25(86.2 \%)$ & $28(82.3 \%)$ & $0.741^{*}$ \\
\hline
\end{tabular}

\#Mean \pm standard deviation by Independent sample $t$ test

$\$$ Median (interquartile range) by Mann-Whitney $U$ test

*Frequency $(\%)$ by chi-square test/Fisher exact test

$p<0.05$ significant

$L K O$ Lucknow, $S G E$ surgical gastroenterology, Uro urology, NS neurosurgery, LAMA left against medical advice was around $25 \mathrm{~km}$ more (13\% more) than the pre-COVID era, though the differences were not statistically significant. The duration of symptoms before reaching the hospital was on an average 6 days in group 2 against 5 days in group 1. Though the most common complaint with which the patients were admitted in both the groups was pain in the abdomen, there was significant decrease in the number of patients with acute abdomen overall ( $82 \%$ vs $58 \%$ with significant decrease in group $2, p$ value 0.039 ). The type of abdominal emergencies was also different. In group 1, the majority of cases included perforation peritonitis, biliary peritonitis following cholecystectomy, necrotizing pancreatitis, liver abscess, or intestinal obstruction necessitating surgical/radiological intervention. In group 2, mild acute pancreatitis, biliary colic, and advanced
Table 2 Distribution of clinical values and interventions between pre and during COVID-19 lockdown $(N=63)$

\begin{tabular}{llll}
\hline Variables & Group 1 $(n=29)$ & Group 2 $(n=34)$ & $p$ value \\
\hline Blood transfusion (\%) & $4(13.8)$ & $6(17.6)$ & 0.741 \\
CAD (\%) & 0 & $1(2.9)$ & 0.999 \\
Vomiting (\%) & 0 & $2(5.8)$ & 0.495 \\
Abdominal pain (\%) & $24(82.8)$ & $20(58.8)$ & 0.039 \\
Headache (\%) & 0 & $7(20.6)$ & 0.041 \\
Hematuria (\%) & $2(6.9)$ & $3(8.8)$ & 0.999 \\
Jaundice $(\%)$ & $3(10.3)$ & $2(5.9)$ & 0.654 \\
DM $(\%)$ & $1(3.4)$ & $6(17.6)$ & 0.112 \\
HTN $(\%)$ & $3(10.3)$ & $4(11.8)$ & 0.999 \\
Imaging done in emergency $(\%)$ & $17(58.6)$ & $22(64.7)$ & 0.795 \\
USG abdomen (\%) & $17(58.6)$ & $13(38.2)$ & 0.106 \\
CT abdomen/head (\%) & $12(41.4)$ & $21(61.8)$ & 0.106 \\
Intervention \{surgery/radiology\} $(\%)$ & $15(65.2)$ & $9(26.4)$ & 0.067 \\
Vaso-pressure support (\%) & $2(6.9)$ & $5(14.7)$ & 0.437 \\
Ventilator $(\%)$ & $2(6.9)$ & $6(17.6)$ & 0.270 \\
Organ support $>$ 48 h (\%) & $2(6.9)$ & $8(23.5)$ & 0.071 \\
\hline
\end{tabular}

Frequency (\%) by Chi-square test/Fisher exact test. $p<0.05$ significant; $D M$ diabetes mellitus, HTN hypertension, $C A D$ coronary artery disease, USG ultrasonography, $C T$ computed tomography 
malignancy with jaundice or ascites were the commonest abdominal emergency. Most of them could be managed conservatively. Two patients required surgery: one underwent nephrectomy for renal cell carcinoma with intractable hematuria, and another underwent percutaneous nephrostomy for obstructive uropathy. A significant increase in the number of patients with neurosurgical emergencies (most commonsubarachnoid hemorrhage) was noted. All patients had severe headache and altered sensorium. They were stabilized, resuscitated, preferably tested for COVID, and taken up for intervention. Two patients required craniotomy and three underwent coiling for aneurysm.

No differences in groups 1 and 2 were recorded in terms of number of patients managed successfully and number of blood transfusion required or mortalities. The major difference noted was in the status of patients at arrival to the ED: more than 4-fold increase in requirement for organ support in group 2 (9 vs 2 in favor of group 2), though the difference was not statistically significant. Six patients with neurosurgical issues required intubation with due precaution. An increase in number of radiological evaluation was noted in group 2. This was because all the patients referred to neurosurgery underwent cross-sectional imaging of the head. The major difference in groups 1 and 2 was the median hospital stay (12 days vs 7 days with significant decrease in group 2, $p=0.007$; Fig. 1).

\section{Discussion}

The COVID-19 pandemic has made a true global footprint and has strained the healthcare facilities across the world including India [7]. Knowledge regarding the presentation and possible management of the COVID infection is improving constantly. New corona hospitals are being set up in every city. The hospital staffs including doctors, nursing personnel, and supporting crew managing these patients are being rotated frequently. The constantly rising number of cases has engaged more than one-third of the workforce in care of the infected.

During the lockdown period, we observed a certain change in the patient load in ED at our center. A minor rise (17\%) in the number of surgical emergencies was noted. The patients had to travel a longer distance (13\% more) to avail the health facilities. The most important difference was increase (4-fold increase) in number of seriously ill patients requiring organ support. Majority of patients with neurological complains required ventilatory support. The increase in requirement of vasopressor support among the patients may be due to various reasons. In pre-lockdown period, the patients were referred to our center by peripheral centers after providing primary care and initial resuscitation, but in the pandemic era, majority of the patients directly came to tertiary care centers. Moreover, the average time to reach the hospital from the start of illness was greater during the lockdown. The health facilities extended by the peripheral private setups remained non-functional due to lack of personal protection equipment and the fear of pandemic in the first month of the lockdown.

In group 2, majority of patients who arrived with abdominal complaints could be managed conservatively. The number of interventions needed was less (26\% vs $65 \%$ ). This was again because majority of the patients came with symptoms of biliary colic or mild pancreatitis which resolved on medications. In normal circumstances, they are usually treated at peripheral centers and need not travel distance to avail health facilities. Further, the strict lockdown also affected the diet habits and social behavior due to isolation that could partially explain the reduction in the incidence of bowel obstruction, severe pancreatitis, and perforation. Similar findings were documented by Patriti et al. in their study [6].

In the pre-lockdown period, many neurosurgical emergencies would be operated in smaller hospitals across the city and the state. In the country, neurosurgery units run in trauma
Fig. 1 Distribution of hospital stay before and during lockdown of COVID-19

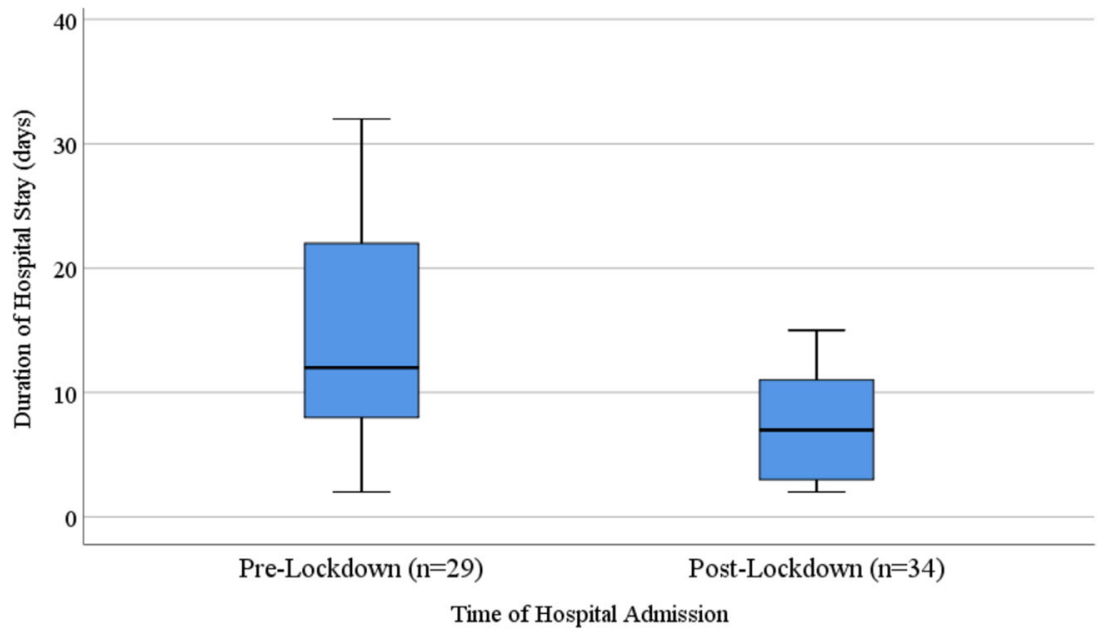


centers which take care of the non-traumatic neurosurgical emergencies as well. With the start of the pandemic, trauma centers in the city were converted into COVID care facilities. This added to the load of neurosurgery cases in the ED which was very visible in the limited duration of the present study. They were managed successfully by the neurosurgery team. At our center, the patients treated in the ED were all low risk for COVID infection admitted after thorough 2 stage screenings (detailed history and thermo scan). None of them tested positive for the virus during the stay. All the patients requiring intervention were first tested for COVID antigen, and those requiring early ventilation were intubated with all due precautions. The overall emergency and hospital stay in group 2 was significantly less as the decision to shift to respective units and intervene were swiftly taken. The upsurge in medical and surgical emergencies necessitated prompt treatment. Moreover, majority of the patients (with mild abdominal complains - biliary colic and mild pancreatitis) improved with conservative management over a short period. The substantial decrease (40\%) in the requirement of intervention was responsible for reduced hospital stay.

The present study points towards the likelihood of upsurge in emergencies presenting to the ED, especially the neurosurgical emergencies. With the ease in travel restrictions, the number of emergencies (including trauma) is set to rise, and this will add to the burden of ED. It is important to make periodic appraisal of the hospital services and the changing patient load in order to apprise the concerned authorities regarding the changing needs. The lacunae need to be highlighted and necessary adjustments made. This will also help to reactivate and coordinate with the local healthcare centers for mutual support and mitigate the undue load on a tertiary care center. The use of referral and counter-referral systems can help to efficiently distribute the work load, provide home care (with the help of local hospitals), and avoid unnecessary admissions in the hospital.

\section{Compliance with Ethical Standards}

Conflict of Interest The authors declare that they have no conflict of interest.

Ethics Approval This study was approved by the Institute's Ethics Committee and patient consent was waived off (IEC code: 2020-134IP-EXP-20).

\section{References}

1. Cao Y, Li Q, Chen J et al (2020) Hospital emergency management plan during the COVID-19 epidemic. Acad Emerg Med 27(4):309311

2. Li Q, Guan X, Wu P, Wang X, Zhou L, Tong Y, Ren R, Leung KSM, Lau EHY, Wong JY, Xing X, Xiang N, Wu Y, Li C, Chen Q, Li D, Liu T, Zhao J, Liu M, Tu W, Chen C, Jin L, Yang R, Wang Q, Zhou S, Wang R, Liu H, Luo Y, Liu Y, Shao G, Li H, Tao Z, Yang Y, Deng Z, Liu B, Ma Z, Zhang Y, Shi G, Lam TTY, Wu JT, Gao GF, Cowling BJ, Yang B, Leung GM, Feng Z (2020) Early transmission dynamics in Wuhan, China, of novel coronavirusinfected pneumonia. N Engl J Med 382(13):1199-1207

3. Hisaka A, Yoshioka H, Hatakeyama H, Sato H, Onouchi Y, Anzai N (2020) Global comparison of changes in the number of test-positive cases and deaths by coronavirus infection (COVID-19) in the world. J Clin Med. 9(6)

4. Ministry of Health and Family Welfare Government of India (2020) Updated Containment Plan for Large Outbreaks Novel Coronavirus Disease 2019 (COVID-19). Retrieved from https://www.mohfw. gov.in/

5. Vadlamudi S, Shanker KS (2020) Lockdown deals deadly blow to kidney patients, The Hindu. Retrieved from https://www.thehindu. com

6. Patriti A, Eugeni E, Guerra F (2020) What happened to surgical emergencies in the era of COVID-19 outbreak? Considerations of surgeons working in an Italian COVID-19 red zone. Updat Surg 72(2):309-310

7. Dong E, Du H, Gardner L. An interactive web-based dashboard to track COVID-19 in real time [published correction appears in Lancet Infect Dis. 2020 Jun 12;:]. Lancet Infect Dis 2020;20(5):533-534

Publisher's Note Springer Nature remains neutral with regard to jurisdictional claims in published maps and institutional affiliations. 